\title{
Increased LEAP Utilization Through Automation of Multi-specimen Alignment and Acquisition
}

David Reinhard, Tim Payne, Eric Strennen, Brian Geiser, Gard Groth and Dan Lenz

CAMECA Instruments, Inc., Madison, Wisconsin, United States

As the interest in atom probe analysis continues to increase in the microelectronics industry, so to do the demands for increased productivity and utilization [1-3]. In response, CAMECA ${ }^{\circledR}$ is introducing several new features that automate LEAP $^{\circledR}$ operation to capture unstaffed analysis time and move atom probe tomography (APT) from a powerful, but niche microscopy towards high-volume testing and manufacturing environments.

The automation is facilitated through a number of new features. First, machine vision makes automatic navigation and rough specimen alignment possible. The project plan allows the acquisition parameters to be defined for a given specimen and tells the instrument how to run the experiment. A chain acquisition module enables the LEAP to run a series of specimens from a microtip array in a user-specified order. To prevent data acquisition with a compromised local electrode after a specimen fracture, an automatic flat test feature can check the viability of an electrode and then continue with the next analysis if it is good, or notify the analyst if a new electrode is needed. The automated alignments, chain acquisition, and automated flat test are designed in conjunction with a new microtip array that positions twenty five specimen mounts such that all specimens can be analyzed without the need to rotate the array in the holder. Some complicated experiments can require changing the acquisition parameters for yield, throughput, or data quality reasons. For these experiments, a scripted acquisition feature allows these parameters to change based on analysis of live data streams rather than manual operator intervention. Changes in acquisition parameters can sometimes depend on accurate analysis of peaks in the mass spectrum (charge state ratios, composition depth profiles, etc.). To ensure good decisions are made, a live calibration continuously updates the voltage, flight path, and mass calibrations.

These features largely automate the process of APT data acquisition thereby expanding the time available for productive use of the LEAP and freeing up staff for more productive activities.

\section{References}

[1] W. Vandervorst et al., Phys. Status Solidi C11 (2014), p. 121.

[2] A.D. Giddings et al., Scr. Mater. 148 (2018), p. 82.

[3] J.P. Barnes et al., Scr. Mater. 148 (2018), p. 91. 